\title{
Rheumatic fever and social justice
}

Alex Brown, Malcolm I McDonald and Tom Calma

High rates of this disease are the face of Indigenous disadvantage

$\mathrm{W}$ hile acute rheumatic fever (ARF) has become a rare curiosity in Australia's non-Indigenous population, its incidence in Indigenous Australians living in remote areas remains among the highest reported in the world. It is unlikely that such a stark contrast between two populations living within the same national borders exists for any other disease or on any other continent.

The new evidence-based review and guideline for diagnosis and management of ARF and rheumatic heart disease (RHD) is an important tool for clinicians who care for Indigenous Australians (page 581). ${ }^{1}$ But the guideline also prescribes a clear course of action for health policymakers. It makes a compelling case for focusing on the provision of secondary prophylaxis via coordinated, register-based RHD control programs that have guaranteed long-term funding. At the minimum, programs are needed in the Top End of the Northern Territory, Central Australia (including areas of South Australia and Western Australia near the NT border), northern WA, and northern Queensland. Other jurisdictions may also require control programs, but further disease burden data are needed to establish this.

It is critically important to ensure that people with ARF and RHD receive good treatment and preventive care. But let's not lose sight of the main game. Treatment of ARF and RHD, and secondary prevention-based control programs, are bandaid solutions to an underlying tragedy.

ARF and RHD are classic diseases of social injustice. The past 50 years have witnessed dramatic declines in the prevalence of ARF and RHD throughout the industrialised world, resulting mainly from improvements in living conditions, socioeconomic conditions, sanitation and medical care, and from reduction in household crowding. ${ }^{2}$ Unfortunately these improvements are yet to be seen among a number of populations defined by socioeconomic status, ethnicity or geographical location. ${ }^{3}$ In essence, ARF and RHD are not only diseases exclusively borne by the disadvantaged, 


\section{EDITORIALS}

but also key indicators of disadvantage itself. In remote Australian Indigenous populations, the effects of disadvantage are so entrenched that it is likely to take several generations, and steadfast political will, before they are overcome.

Rates of death from RHD among Aboriginal people in the Top End of the NT exceed those reported in many industrialised countries over a century ago. ${ }^{4}$ There is little or no evidence of improvement over at least the past three decades. ${ }^{5}$ As a consequence, Indigenous Australians continue to die before their time from a highly preventable, highly treatable and completely avoidable illness. ${ }^{4}$

Ongoing disparities in the burden of ARF and RHD reflect a number of failures in the development and delivery of health and health-related services. Failure to provide secondary prophylaxis can be due to missed diagnoses, poor continuity of care, a lack of trust and communication between patients and care providers, high staff turnover, a lack of appropriate health education, and, perhaps most importantly, a lack of political and bureaucratic commitment to solving the problem. Failure in primary prevention of ARF and RHD (ie, in preventing the acquisition of group A streptococcal infections) reflects the failure to provide Indigenous communities with the appropriate type and level of housing and environmental conditions that all Australians should expect.

The federal government has targeted a 50\% reduction in death due to RHD by $2008 .{ }^{6}$ While this is an unrealistic expectation, significant achievements are possible within the short to medium term, as has been witnessed with comprehensive approaches to control of ARF and RHD in the French Caribbean ${ }^{7}$ and with the establishment of register-based ARF/RHD control programs across New Zealand. ${ }^{8}$ Perhaps the most frustrating thing is that preventing premature death due to RHD is more achievable than solving an ever expanding list of other health and social problems facing Indigenous Australians.

To prevent premature death due to RHD, a number of concrete steps must be taken. Firstly, we must commit to alleviating the underlying socioeconomic determinants of ARF and RHD. The most important of these determinants — overcrowded housing is also the easiest to address, but requires a dramatically greater investment by governments than we are currently seeing. A recent study confirmed the extreme levels of household crowding experienced in many remote communities: ${ }^{9}$ in two large NT Aboriginal communities, the median number of people per house was 17 and 14 , respectively, with a median of 6.9 and 7.5 people per bedroom, respectively. Secondly, we must ensure that each person with a history of ARF or RHD receives appropriate care. This entirely achievable goal would prevent Aboriginal children dying unnecessarily from this disease. Thirdly, we must ensure that political will delivers the deliverable and prevents the preventable.

As long as modern Australia continues to accept the large and growing health and social disparities experienced by its Indigenous people, it fails in its duty to protect and provide for the most vulnerable. Will we be brought to account for our failure to deal with these disparities, or will cries for justice be silenced, as has happened with so much of the history of Australia's first people?
1 Centre for Indigenous Vascular Research, Baker Heart Research Institute, Alice Springs, NT.

2 Menzies School of Health Research, Institute of Advanced Studies, Charles Darwin University, Darwin, NT.

3 Human Rights and Equal Opportunities Commission, Sydney, NSW.

Correspondence: alex.brown@baker.edu.au

\section{References}

1 Carapetis JR, Brown A, Wilson NJ, Edwards KN, on behalf of the Rheumatic Fever Guidelines Writing Group. An Australian guideline for rheumatic fever and rheumatic heart disease: an abridged outline. Med J Aust 2007; 186: 581-586.

2 Quinn R. Comprehensive review of morbidity and mortality trends for rheumatic fever, streptococcal disease, and scarlet fever: the decline of rheumatic fever. Rev Infect Dis 1989; 11: 928-953.

3 Carapetis JR, Steer AC, Mulholland EK, Weber M. The global burden of group A streptococcal diseases. Lancet Infect Dis 2005; 5: 685-694.

4 Carapetis JR, Currie BJ. Mortality due to acute rheumatic fever and rheumatic heart disease in the Northern Territory: a preventable cause of death in aboriginal people. Aust N Z J Public Health 1999; 23: 159-163.

5 Carapetis JR, Wolff DR, Currie BJ. Acute rheumatic fever and rheumatic heart disease in the Top End of Australia's Northern Territory. Med J Aust 1996; 164: 146-149.

6 Office of Aboriginal and Torres Strait Islander Health. National performance indicators and targets for 1998-2000 to monitor governments' efforts to improve Aboriginal and Torres Strait Islander health. Canberra: OATSIH, Commonwealth Department of Health and Aged Care, 1998.

7 Bach J, Chalons S, Forier E, et al. 10-year educational programme aimed at rheumatic fever in two French Caribbean islands. Lancet 1996; 347: 644-648.

8 Thornley C, McNichols A, Baker M, Lennon D. Rheumatic fever registers in New Zealand. N Z Public Health Rep 2001; 8: 41-44.

9 McDonald MI, Towers RJ, Andrews RM, et al. Low rates of streptococcal pharyngitis and high rates of pyoderma in Australian aboriginal communities where acute rheumatic fever is hyperendemic. Clin Infect Dis 2006; 43: 683-689

\section{Author details}

Alex Brown, BMed, MPH, FCSANZ, Head ${ }^{1}$

Malcolm I McDonald, FRACP, Senior Research Fellow ${ }^{2}$

Tom Calma, Aboriginal and Torres Strait Islander Social Justice

Commissioner $^{3}$ 